\title{
EINE KOLUMBIANISCHE GRABURNE ZUR AUSSTELLUNG IM VÖLKERKUNDEMUSEUM DER UNIVERSITÄT ZÜRICH
}

\author{
Hans TanNer
}

Aus dem kolumbianischen Magdalenatal ist seit dem Anfang der Dreißigerjahre eine Anzahl von Urnentunden bekannt geworden, die von G. REICHEL-DoLMATOFF 11943 erstmals zusammenfassend bearbeitet wurden. Von 12 verschiedenen Fundstellen, die sich auf das untere und das mittlere Magdalenatal verteilen, werden einander in der Form verwandte Graburnen beschrieben, deren Deckel teils mit anthropomorphen, teils mit zoomorphen Figuren geschmückt sind.

Besonders wichtige Funde stammen aus dem Tal des Rio Miel, dem Grenzfluß zwischen den Departementen Caldas und Antioquia, von wo eine solche Menge Grabkeramik und Steinbeile beschrieben ist, daß man annehmen muß, hier habe eine ständige stärkere Besiedlung geherrscht. Hier lebten zur. Zeit der Eroberung des Landes durch die Spanier die Pantagora- oder PalenqueIndianer, die nach P. River ${ }^{2}$ zwischen die Ost- und die Westgruppe der kolumbianischen KaribIndianer zu stellen sind, wenn auch ihre Zugehörigkeit zu den Kariben noch umstritten ist. Anthropomorphe Figuren auf Urnendeckeln vom Rio Miel, wie auch von andern von ReICHELDolmatofF beschriebenen Fundstellen, weisen des öfteren Einschnürungsdeformationen am Oberarm und am Unterschenkel unterhalb des Knies und oberhalb des Knöchels auf, die nach River (op. cit.) typisch sein sollen für Karib-Indianer, oder für Stämme, die in engem Kontakt mit diesen lebten.

Heute leben wohl nur noch spärliche Reste der ursprünglichen Indianerbevölkerung in den Wäldern des mittleren Magdalenatals und in Rückzugsgebieten in der benachbarten Ostkordillere. So schreibt EUGSTER ${ }^{3}$, daß vor etwa 30 Jahren im Waldgebiet zwischen Rio Opón und Rio Carare noch etwa 20 männliche Stammesangehörige der Opones lebten, die einen Karibendialekt sprachen. Sie durchzogen als primitive nomadisierende Jäger die Wälder und waren damals wegen ihrer Raubüberfälle auf die kolumbianischen Siedler sehr gefürchtet. Während meines mehr als einjährigen Aufenthaltes in den Urwaldgebieten des benachbarten Territorio Vasquez in den Jahren $1951 / 52$ sind mir allerdings keine Spuren von lebenden Indianern mehr zu Gesicht gekommen.

Trotz der recht zahlreichen Urnenfunde (im kolumbianischen Nationalmuseum in Bogotá befindet sich eine umfangreiche Sammlung) fehlt aber bis heute meines Wissens jede Beschreibung eines Grabes. Das liegt wohl z. T. darin begründet, daß in Kolumbien seit der Conquista der Grabraub sehr verbreitet war, die Grabräuber sich aber kaum für Form und Aussehen der Gräber, sondern nur für deren Inhalt interessierten. Ein Teil der Urnenbestände in den Museen mag aus derartig ausgeraubten Gräbern stammen, während andere Fundstücke durch die durch die Erosion bedingte Zerstörung von Gräbern ans Tageslicht gelangt sein mögen.

Bereits 1938 hatte Gregorio Hernandez de Alba ${ }^{*}$ einige dieser Urnen beschrieben und sie als zur „civilizacion Moskito" gehörend bezeichnet, weil der Großteil der ihm zur Verfügung stehenden Untersuchungsstücke auf der Hacienda Moskito in der Gegend von Ocaña gefunden worden war. Auch BENNETT beschäftigt sich im "Handbook of South American Indians " 5 in einem kurzen Abschnitt mit der „Moskitokultur“. Dieser Name ist aber recht unglücklich gewählt, denn er könnte leicht zu Verwechslungen und unerwünschten Hinweisen auf die in Zentralamerika lebenden Mosquitoindianer führen. Im „Museo Nacional“ in Bogotá wird deshalb seit einer Reihe von Jahren an seiner Stelle die Bezeichnung "cultura del valle Magdalena“ verwendet. Im übrigen ist von dieser „Kultur“ außer den Graburnen, einigen weiteren aus Gräbern stammenden Töpfen, Keramikgegenständen und Steinbeilen und einigen wenigen Töpfen für den Hausgebrauch bisher nichts beschrieben worden.

Den Anlaß für die vorliegende kurze Studie bildet die im Oktober 1951 erfolgte Auffindung eines gut erhaltenen Grabes dieser "Magdalenatalkultur" durch Arbeiter der Texas Petroleum Company im dieser Ölgesellschaft gehörenden Territorio Vasquez in der Nähe von Pto. Niño im Departemente Boyacá. Von der Direktion des „Museo Nacional“ in Bogotá mit der bestmöglichen Bergung der Funde und der Vermessung des Grabes betraut, konnte ich die Erlaubnis erlangen, eine der 14 Urnen samt Deckel ins Ausland mitzunehmen und sie der Sammlung für Völkerkunde der Universität Zürich zu übergeben. Im folgenden möchte ich eine Beschreibung dieser Urne, einen kurzen Überblick über die andern Fundgegenstände, sowie erstmals Pläne dieses Grabes veröffentlichen. Eine ausführliche Bearbeitung der Fundgegenstände ist für später im Rahmen einer vom kolumbianischen Nationalmuseum herauszugebenden Publikation vorgesehen. Dort sollen auch die genauen Koordinaten des Grabes gegeben werden.

Die Entdeckung des Grabes in dem mit dichtem tropischen Urwald bewachsenen Territorio Vasquez ist einem glücklichen Zufall zu verdanken. Bei Bauarbeiten an der zur Bohrstelle Boyacá-1 führenden Straße wurde von einem Caterpillar-Traktor beim Abtragen eines Hügels die Grabstelle teilweise freigelegt. Die kolumbianischen Arbeiter machten sich sofort an die Ausräumung des Grabes, sodaß im Augenblicke meines Eintreffens auf der Fundstelle - etwa 15 Minuten später 
- das Grab bereits bis auf wenige Reststücke leer war, und die Fundgegenstände in der Nähe im Freien aufgeschichtet lagen. Einzelne Urnen waren dabei von den ins Schatzsucherfieber geratenen Entdeckern umgestülpt worden, sodaß ihr. Knocheninhalt auf dem Boden ausgebreitet lag, und eine anthropomorphe Deckelfigur war gar entzwei gebrochen, weil in ihrem hohlen Innern Tonkugeln gerasselt hatten und die Arbeiter deshalb Smaragde zu finden hofften.
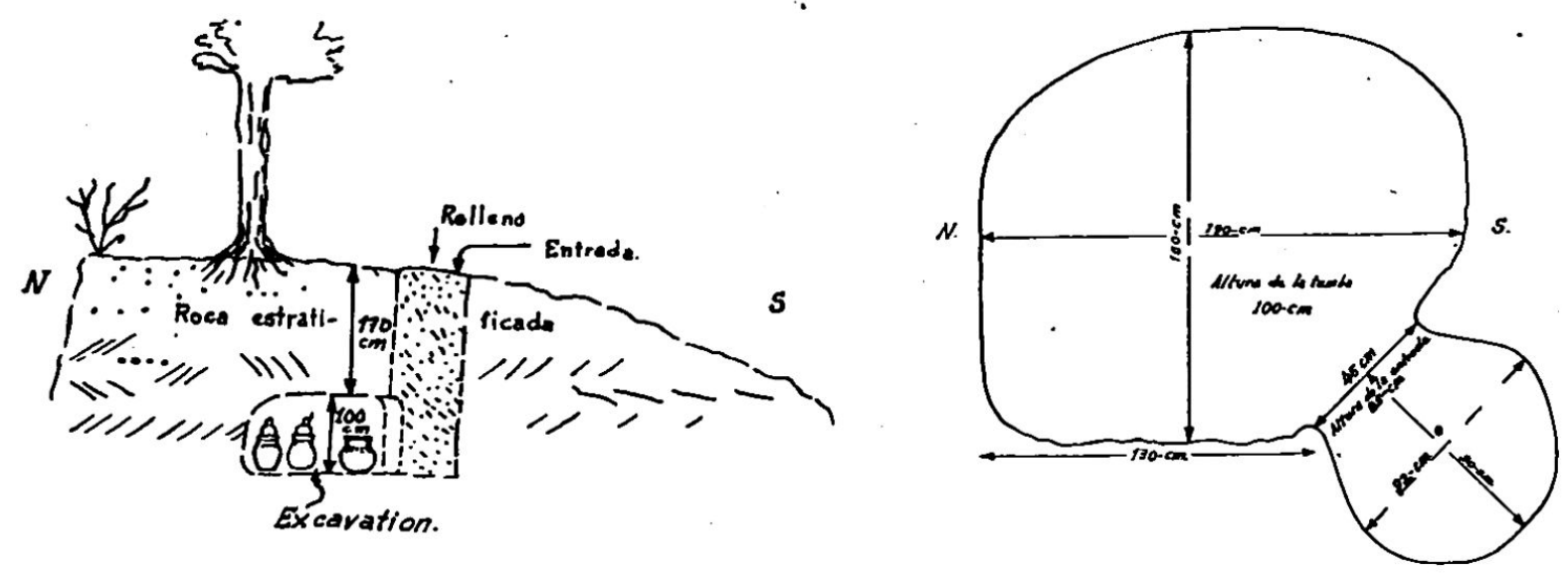

Abb. 1 Grundriß und Aufriß des Grabes. Relleno und Entrada: Mit Sand gefüllter Eingangsschacht. Roca ostratificada: Anstehender Sandstein. Excavation: Grabkammer. Altura de la tumba: Höhe des Grabes. Altura de la entrada: Höhe der Eingangspforte.

Das Grab hatte unter der Einwirkung des Traktors nur wenig gelitten und konnte leicht rekonstruiert werden; hingegen war es wegen der vorangegangenen Erdbewegungen nicht mehr möglich, festzustellen, ob auf der ehemaligen Hügeloberfläche Hinweise auf das Vorhandensein der Grabstätte zu finden gewesen wären. Aus diesen Gründen sind einzelne der im folgenden gemachten Ausführungen unvollständig, während sich andere nicht auf eigene Beobachtungen, sondern auf die - allerdings übereinstimmenden - Aussagen der Arbeiter, die von mir kurz nach meinem Eintreffen einzeln befragt worden waren, stützen.

Die Grabstelle befindet sich auf dem Gipfel eines bewaldeten Hügels, der sich in keiner Weise von der Menge der anderen niederen Hügel dieser Gegend unterscheidet, und liegt etwa $10 \mathrm{~km}$ vom Magdalenastrom (auf dem rechten Ufer) und etwa $1 \mathrm{~km}$ vom nächsten größeren Bach, der Quebrada Vasquez, entfernt. Die Hügelkuppe erhebt sich etwa $10 \mathrm{~m}$ über die größtteils versumpfte Talsohle. Von der Hügeloberfläche führt ein Eingangsschacht $(90 \times 92 \mathrm{~cm}$ weit $)$ durch pleistocaenen, gut verkitteten-konglomeratführenden Sandstein mit deutlich ausgeprägter Kreuzschichtung $270 \mathrm{~cm}$ tief senkrecht nach unten (Abb. 1). Durch eine $45 \mathrm{~cm}$ breite und $85 \mathrm{~cm}$ hohe Eingangspforte wird eine künstlich ausgehöhlte Grabkammer erreicht, deren größte Länge $190 \mathrm{~cm}$, größte Breite $160 \mathrm{~cm}$ und größte Höhe $100 \mathrm{~cm}$ ist. Diese Grabkammer war bei der Entdeckung außerordentlich gut erhalten, sie zeigte an Wänden und Decke noch deutlich die Hauspuren ihrer Ersteller. Der Eingangsschacht dagegen war mit Sand gefüllt, der nach Unterbringung der Urnen im Grabe offenbar sehr sorgfältig und wohl in nassem Zustande eingeführt worden war, denn nach meinen Feststellungen ist kein Sand durch die Eingangspforte ins Grab gekollert. Spuren einer Absperrung der Grabhöhle gegen den Schacht hin, sei es durch Steine oder Holzsperren, konnte ich keine finden.

In der Nord-Süd orientierten Grabkammer fanden sich 14 zum größten Teil wohl erhaltene Graburnen, deren Deckel sämtliche mit anthropomorphen Figuren geschmückt waren. Nach Aussagen der kolumbianischen Arbeiter schauten alle diese Figuren nach Norden.

Die vierzehn zum Teil sehr formschönen Urnen sind alle aus gebranntem Ton hergestellt, der, wie an Bruchstücken ersichtlich ist, stellenweise mit Silt und feinem Sand verunreinigt ist. Sie sind hellgrau-braun bis rotbraun und stellenweise mit schwarzen Flecken besetzt. Spuren von Bemalung konnte ich keine feststellen. Die Urnen lassen sich in zwei Formgruppen aufteilen, wobei aber die einzelnen Angehörigen dieser Gruppen unter sich noch recht verschieden sind. Gruppe I umfaßt 9 Urnen von 29 bis $48 \mathrm{~cm}$ Höhe und 26 bis $38 \mathrm{~cm}$ größter Breite. Sie machen alle einen hohen schlanken Eindruck (Abb. 2), während die 5 Urnen der Gruppe II, die zwischen 33 und $40 \mathrm{~cm}$ hoch und bis $40 \mathrm{~cm}$ breit sind, weit gedrungener wirken (Abb. 4 und 5). Alle Urnen sind mit geometrischen Figuren geschmückt, die auf den Gefäßen der Gruppe I (mit einer Ausnahme) in zwei auf entgegenge- 


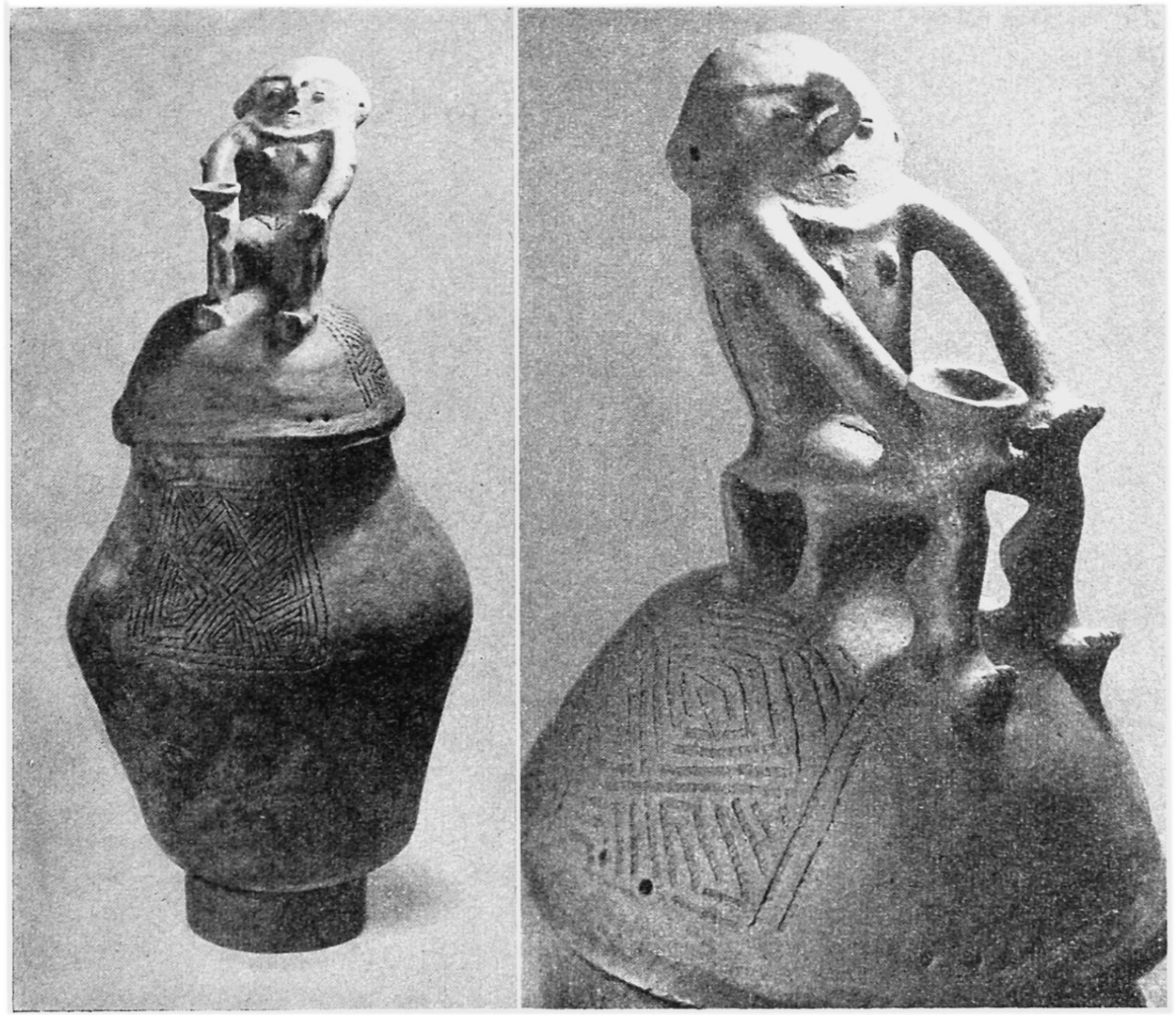

Abb. 2 (links) Graburne der Gruppe I (Höhe $41 \mathrm{~cm}$ )

Abb. 3 (rechts) Deckel mit sitzender anthropomorpher Figur (Höhe des Deckels $11 \mathrm{~cm}$, der Figur $21 \mathrm{~cm}$ ). Ausgestellt in der Sammlung für Völkerkunde der Universität Zürich. Fotos Dr. EisY LEUZiNGER

setzten Seiten liegenden einfachen, meist trapezförmigen Ornamentsflächen angeordnet sind, wobei bei einzelnen Urnen die Ornamente der Vorder- und Rückseite stark voneinander abweichen. Die Verzierungen bestehen aus meist geraden, seltener leicht gekurvten vertieften Linien und zu Linien angeordneten, oder auch anscheinend wahllos hingestreuten vertieften Punkten. Linien und Punkte wurden mit einem stumpfen Instrument in den noch weichen Ton eingestoßen, wie besonders schön an der im Zürcher Museum ausgestellten Urne zu sehen ist. Die Ornamente der Gruppe II dagegen laufen als zusammenhängendes Muster rund um den Hals der Urnen. Sie sind im allgemeinen auch sorgfältiger und komplizierter ausgeführt und weisen neben den einfachen vertieften Linien auch vorspringende Borden und in einem Fall zu Vierergruppen angeordnete Warzen auf der Schulter des Gefäßes auf (Abb. 4).

Ähnliche einfache Strichmuster wiederholen sich auf einzelnen Urnendeckeln (Abb. 3). Alle Urnendeckel aber sind von sitzenden anthropomorphen Figuren gekrönt, die in Form und Ausführung weitgehend miteinander verwandt sind. Zwar haben einzelne Figuren eckige, trapezförmige Köpfe (Abb. 6), während die Mehrzahl eine gerundete Kopfform aufweist. Auch sind beide Geschlechter vertreten. 3 Figuren zeigen deutlich männliche Geschlechtsmerkmale, 8 sind weiblich, während 2 Deckel zu stark zerstört sind für eine Geschlechtsbestimmung. Aber allen Figu- 

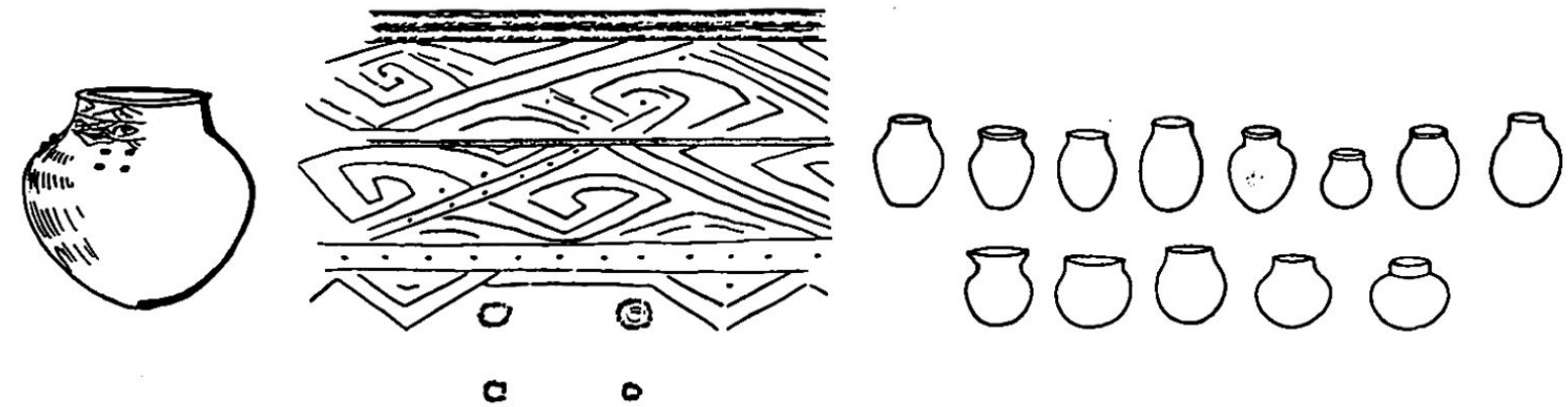

Abb. 4 (links) Graburne der Gruppe II (Höhe $36 \mathrm{~cm}$, größte Breite $40 \mathrm{~cm}$, Durchmesser der Öffnung $20 \mathrm{~cm}$ ). Mitte; Ausschnitt aus dem rund um den Urnenhals laufenden Ornament.

Abb. 5 Uberblick über die im Grabe gefundenen Urnenformen. Oben Gruppe I, unten Gruppe II.

ren ist die starre, aufrechte Haltung eigen, alle sitzen mit geöffneten Beinen nach rückwärts gelehnt und stützen die Hände auf die Knie. Alle stimmen auch in der stark stilisierten, ringförmigen $\mathrm{Nase}$, in den vorspringenden Augen, im kleinen, vertieften Mund und in den nicht vom Kopf abgetrennten Ohren mit durchbohrten Ohrlöchern überein. Bei der Mehrzahl der Köpfe ist der in die Stirne fallende Haarwuchs durch ein Vorspringen des Kopf-Oberteils angedeutet, bei einer Figur dagegen sind nur vorspringende Augenbraunen zu sehen. Der Kopf ruht bei fast allen Gestalten unmittelbar auf dem Rumpf; nur bei einer Figur ist ein Hals angedeutet (Abb. 7). Dagegen sind bei allen Statuetten ein bis zwei 《Kinnwülste» vorhanden. Vorstehende Brustwarzen, bei einzelnen Figuren ein Nabelloch, vorstehende oder eingekritzte Geschlechtsorgane, und bei der Mehrzahl ein deutlich vorspringendes Rückgrat, dazu zwei nur hie und da angedeutete, im allgemeinen aber offene Schlitze, auf den Lendenseiten fallen bei einer Betrachtung des Rumpfes auf, der im übrigen bei einigen Figuren in seinem hohlen Innern mehrere Tonkugeln enthält, die beim Hin- und Her-Bewegen der Urnendeckel ein rasselndes Geräusch verursachen. Die nach auswärts gerichteten, auf die Knie aufgestützten Arme erscheinen gegenüber dem Rumpf als zu lang, ebenso die wagrecht nach vorn gestreckten Oberschenkel. Die senkrecht nach unten gerichteten Unterschenkel dagegen scheinen etwas verkürzt und zeigen durchwegs eine starke Einschnürung unter dem Knie und stark übertrieben ausgebildete Waden, also nach Rivet eine typische «Karibdeformation». Deformierte und eingeschnürte Oberarme dagegen konnte ich nur bei einem kleinen 'Teil der Statuetten feststellen (Abb. 7). Bei allen Figuren fällt die eigenartige Fußstellung und der stark vorspringende Fußknöchel auf. Dieser wie auch der vorspringende Ellbogen sind bei einzelnen Gestalten nachträglich aufgesetzt. Finger und Zehen sind meist nur angedeutet, was auch darin zum Ausdruck kommt, daß das eine Mal nur vier, das andere Mal aber fünf oder sechs Finger oder Zehen vorhanden sind. Schließlich tragen alle Figuren (mit der Ausnahme eines Linkshänders) in der rechten Hand ein schalenförmiges Gefäß. Spuren einer Bemalung konnte ich u. a. auf einer Figur feststellen, wo vom Mund zu den beiden Ohrlöchern hin eine schwach angedeutete schwarze Doppellinie führt, während sich über Brust und Bauch ein in der Nabelgegend verzweigender, stärker ausgeprägter schwarzer Streifen zieht (Abb. 6). Außer einer einfachen Lochreihe auf der Oberkante des Kopfes bei einer Figur habe ich keine weitern Dekorationen an den Statuetten selbst gefunden.

Die meisten Figuren sitzen auf einfachen Hockern; bei zweien dient aber eine vierbeinige zoomorphe Figur, deren Kopf nach links und Schwanzstummel nach rechts gerichtet ist, als Sitzgelegenheit. Die Identifizierung dieser Tierfiguren bereitet einige Schwierigkeiten. Man könnte am ehesten an Frösche denken, wie sie u. a. auch auf Urnen vom Rio Miel gefunden wurden, doch wollen die Schwanz- 
stummel nicht recht zum Bilde eines Frosches passen. Eine Figur gleicht einer Schildkröte.

Am untern Rand haben alle Deckel eine Anzahl - meist vier - Lochpaare, durch die wohl seinerzeit Schnüre durchgezogen worden waren, um die Deckel auf den Urnen $z u$ befestigen. Von diesen Schnüren waren allerdings keine Überreste mehr zu finden. Die Urnendeckel sind zwischen 25 und $34 \mathrm{~cm}$ hoch, die Figuren erreichen eine Höhe von $15 \frac{1}{2}$ bis $25 \mathrm{~cm}$.
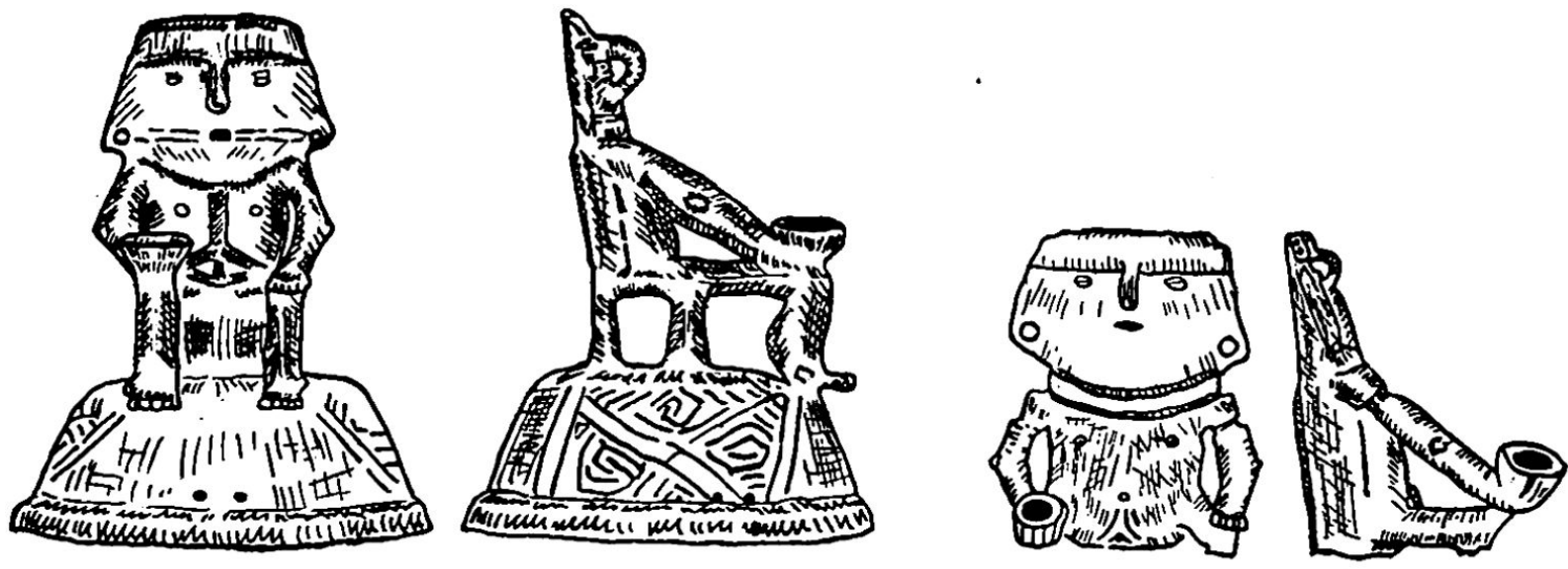

Abb. 6 (links) Deckelfigur mit trapezförmigem Kopf. Auf Gesicht und Rumpf Spuren einer schwarzen Bemalung. Gesamthöhe $30,5 \mathrm{~cm}$.

Abb. 7 (rechts) Fraginent einer männlichen Deckelfigur mit Lochverzierungen auf der Kopfoberkante. Deutlich ausgeprägter Hals und gut sichtbare Einschnürungsdeformationen an den Oberarmen.

In den Urnen drin (nach Aussagen der Arbeiter in allen Urnen) fanden sich zum Teil stark mitgenommene Knochen, die bei der etwas rauhen «Bergung 》 durch die Arbeiter, die ja in den Urnen nach Schätzen suchten, fast alle in Stücke gingen. Immerhin war ein gut erhaltener menschlicher Unterkiefer, ein prachtvoll erhaltener Schädel mit noch unverknöcherter Lambdanaht, ein Schädel, dem der Gesichtsteil tehlte (ebenfalls mit unverknöcherten Nähten) und verschiedene Röhrenknochen, außerdem eine große Anzahl gut erhaltener Zähne vorhanden. Es scheint außer $Z$ weifel zu sein, daß es sich bei dieser Fundstelle um ein Sekundärgrab handelt, daß also die Toten wohl irgendwo in der Erde zum ersten Mal begraben, nach einiger Zeit aber wieder ausgegraben und die Knochen aufs neue, diesmal in Graburnen, beigesetzt worden waren*.

In der Zürcherurne fand sich außerdem ein kleines, anscheinend nicht bearbeitetes Silexstück. Steinbeile sind von andern Fundstellen eine ganze Reihe bekannt (Reichel-Dolmatoff, op. cit.). Eigenartig ist nur, daß sich das Fundstück in der Urne selbst und nicht auf den Boden des Grabes befand.

Neben diesen Urnen waren im Grabe noch zwei kleine, je $17 \mathrm{~cm}$ hohe Tonschalen, die mit einfachsten Strichmustern verziert sind. Möglicherweise haben diese Schalen dazu gedient, den Toten Nahrung ins Grab zu geben, und es ist wohl anzunehmen, daß auch die Gefäße, die die Deckelfiguren in ihren Händen halten,

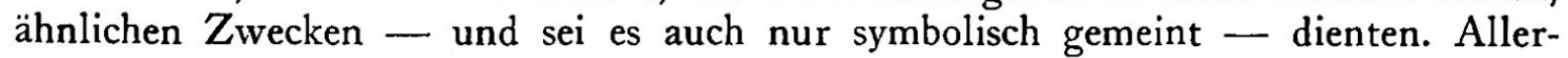

* Leider wurde mir der besterhaltene Schädel schon auf der Fundstelle entwendet. Die übrigen noch einigermaßen gut erhaltenen Knochenreste habe ich dem Nationalmuseum in Bogotá übergeben. In der im Zürcher Museum ausgestellten Urne befindet sich eine große Menge von Knochenfragmenten. Stücke davon wurden von Herrn Prof. F. SchWarZ vom gerichtsmedizinischen Institut der Universität Zürich untersucht, wofür ich ihm auch an dieser Stelle meinen herzlichsten Dank aussprechen möchte. Auch seine Untersuchungen ergaben, daß diese Knochenbruchstücke keine Kremationsspuren aufweisen. 
dings zeigen die Töpfe in Innern gar keine Verunreinigungen von allfälligen Nahrungsüberresten.

Mit 12 geweißten, durchlochten Tonscheibchen von je ungefähr $2 \mathrm{~cm}$ Durchmesser, die auf dem Boden des Grabes lagen, vervollständigt sich die Fundliste. Auf den ersten Blick scheint es sich um Teile einer Halskette zu handeln; es ist aber wohl möglich, daß diese Scheibchen einzelnen Deckelfiguren auf die vorstehenden Augen aufgesetzt gewesen waren, denn wir kennen aus dem Rio Miel und von andern Fundstellen Grabfiguren, deren Augen mit derartigen Plättchen bedeckt sind (Reichel-Dolmatoff op. cit.). Allerdings sind bei jenen Figuren auch Kopf, Rumpf und Gliedmaßen mit kleinen, weißen durchlochten Scheibchen beklebt, die aber, zum Unterschied von denen in unserm Grabe gefundenen, nicht aus gebranntem Ton, sondern aus Fischbein bestehen.

Wenn wir die im Vorhergehenden besprochenen Fundgegenständen mit jenen von andern Lokalitäten vergleichen, so fällt sofort eine weitgehende Verwandtschaft mit Funden vom Rio Miel, der auch geographisch am nächsten liegenden Fundstelle auf. Allerdings sind Urnen und Deckelfiguren vom Rio Miel im allgemeinen sorgfältiger ausgeführt und reichhaltiger dekoriert; aber die Übereinstimmung in Urnenform, in Form und Haltung einzelner Deckelfiguren wie auch in der Ausführung der geometrischen Strichmuster auf Urnen und Deckeln ist in die Augen springend, soda $\beta$ die Vermutung nahe liegt, da $\beta$ die Grabstelle im Territorio Vasquez ebenfalls von den am Rio Miel lebenden Palenque-Indianern oder doch von deren nahen Verwandten angelegt wurde.

\section{LITERATUURACHWE IS}

1 Gérard Reichel-Dolmatoff y Alicia Dussan de Reichel: Las urnas funerarias en la cuenca del Rio Magdalena. Revista del Instituto Etnologico Nacional, Bogotá, t. 1, 1943, p. 209-260. 2 Paul Rivet: La influencia karib en Colombia. Revista del Instituto Etnologica Nacional, Bogotá, t. 1, fasc. 1, 1943, p. 55-93. - 3 Dr. H. EugsTER: Über ethnographische Beobachtungen in Columbien, S.Amerika. In Mitt. Geogr. Ethnogr. Ges. Zürich. Bd. XXIV, 1923/24. - 4 GREgorio Hernandez de Alba: Colombia. Compendio arqueologico. Bogotá, 1938. - 5 Wendell C. BennetT: Archaeology of Colombia, in Handbook of South American Indians, Volume 2, The Andean Civilizations, 1946.

\section{UNE URNE FUNÉRAIRE DE COLOMBIE}

Depuis une série d'années, on trouve dans la vallée de la Magdalena, en Colombie, des urnes funéraires qu'on attribue aux Indiens Caraibes. Le couvercle en est orné de figures fortement stylisées d'hommes ou d'animaux. On décrit ici ces urnes, les figures des couvercles et d'autres accessoires funéraires, et on donne en outre pour la première fois le plan d'un tombeau de cette civilisation colombienne connue sous le nom de «coltura del Valle Magdalena "et aujourd'hui éteinte.

\section{UN'URNA SEPOLCRALE COLUMBIANA}

Nella Valle Magdalena nella Columbia si sono ritrovate, da una serie di anni, urne sepolcrali attribuite agli Indiani Karib. Il loro coperchio è decorato con figure antropomorfe e zoomorfe sensibilmente stilizzate. L'autore descrive queste urne, i loro coperchi e altri oggetti deposti nelle tombe e pubblicca per la prima volta i piani di una tomba dei Sekder, che risale alla „coltura della Valle Magdalena “, oggi estinta.

\section{GEOGRAPHIE UND KARTOGRAPHIE}

AN DER JAHRESVERSAMMLUNG DER SCHWEIZERISCHEN NATURFORSCHENDEN GESELLSCHAFT VOM 22.-25. AUGUST 1952 IN BERN

Beschlußgemäß wurde die 131. Jahresversammlung der S. N. G. in Bern abgehalten. Die Vorträge der Sektion für Geographie und Kartographie, deren Zahl bedauerlicherweise trotz wiederholter Appelle des Zentralpräsidenten, PD. Dr. HANs 